\title{
HARMONIC MAPS TO SPHERES
}

\author{
BRUCE SOLOMON
}

\section{Introduction}

Let $M^{k}$ be an open Riemannian manifold. Let $S^{n} \subset \mathbf{R}^{n+1}$ be the familiar unit sphere. Here $k$ and $n$ are unrestricted positive integers, and for the rest of this paper, $\Sigma=\Sigma^{n-2} \subset S^{n}$ will denote an arbitrarily chosen, totally geodesic subsphere of codimension two. Our principal objects of study will be harmonic maps of the form $F: M \rightarrow S^{n}$, which avoid $\Sigma$. We have discovered that such maps possess special properties.

For example (leaving definitions momentarily aside), if $M$ is compact, $F(M) \cap \Sigma=\varnothing$, and $F$ is null-homotopic as a map to $S^{n} \sim \Sigma$, then $F$ is constant (Theorem 1). When $F$ is energy-minimizing, and bounded away from $\Sigma$ ( $M$ typically noncompact), we obtain regularity and Liouville theorems. Namely, $F$ is everywhere smooth (Theorem 2), and if $M=\mathbf{R}^{k}, n>1$, actually constant (Theorem 4). Note these last two results are false without the boundedness assumption; e.g., the radial projection $\mathbf{R}^{n+1} \rightarrow S^{n}$ minimizes energy whenever $n>6$ (and possibly even when $n>2$ ) [8].

Our paper concludes with an appendix, containing a theorem on nodal (zero) sets of eigenfunctions on a compact Riemannian manifold. We include it here because it leads to an alternate proof of Theorem 1 , and thereby casts a different, more geometric light on our results.

Let us make some of our terminology more precise. For further details, and usage not covered here, we recommend that the reader consult [3] or [7].

Consider a smooth map $F: M^{k} \rightarrow N^{n}$ between Riemannian manifolds, which has square summable first derivatives; that is, $F \in L_{1, \text { loc }}^{2}(M, N)$. Associated to $F$, there is a function on $M$ known as the energy density, and denoted here by $|D F|^{2}$. It is defined, at any point $x \in M$ by the formula

$$
|D F|^{2}=\sum_{i=1}^{k}\left\langle D F\left(e_{i}\right), D F\left(e_{i}\right)\right\rangle_{N},
$$


where $D F$ is the usual differential of $F, e_{1}, \cdots, e_{k}$ is an orthonormal basis for $T_{x} M$, and $\langle,\rangle_{N}$ is the metric on $N$. Integrating the energy density against the volume form $d v_{M}$ on a compact subset $K \subset M$, we obtain a functional known as the energy integral:

$$
\mathbf{E}_{K}(F)=\int_{K}|D F|^{2} d v_{M}
$$

The map $F$ will be termed harmonic if for every compact $K \subset M$, it is a smooth critical point for $\mathbf{E}_{K}$, in the sense of the classical calculus of variations.

An alternative variational hypothesis to harmonicity is that of energy minimization. $F$ is said to be energy-minimizing if

$$
\mathbf{E}_{K}(F) \leqslant \mathbf{E}_{K}(G),
$$

whenever $K \subset M$ is compact, $G \in L_{1, \text { loc }}^{2}(M, N)$, and $G=F$ a.e. on $M \sim K$.

A deep result concerning energy-minimizing maps is the regularity theorem of Schoen \& Uhlenbeck [7, Theorem II], which states that such maps are always smooth on the complement of a closed subset of $M$ (the singular set of $F$, denoted $\operatorname{sing}(F)$ ), whose Hausdorff codimension is at least 3 . The same authors, in a subsequent paper [8] studied the special case $N=S^{n}$, showing that $\operatorname{sing}(F) \subset M$ is then generally smaller, having codimension at least $C(n)$. $C(n)$ is an explicitly given integer which depends on $n$, but is always less than 8. (For $k<C(n)$, this of course implies global regularity.) They then deduce a Liouville theorem, corresponding to their smoothness result, under the dimensional restrictions $k<C(n), n \geqslant 3$. Namely, for such $k$ and $n$, an energyminimizing map $F: \mathbf{R}^{k} \rightarrow S^{n}$ is constant.

By way of comparison, the regularity and Liouville theorems we obtain in this paper are analogous to the latter results of [8], replacing the dimensional restrictions there with the condition that $F$ be bounded away from $\Sigma$, as mentioned earlier.

The salient feature of $S^{n} \sim \Sigma$ here is its warped product structure, as discussed in $\S \S 1,2$. In fact, our smoothness argument, which is based on the results of Schoen \& Uhlenbeck in [7], goes through in this more general, warped product setting. As a consequence, we are able to deduce regularity for a larger class of targets than in [7] (Theorem 3).

In addition to our main results, we obtain several corollaries. Among other things, these deal with minimal submanifolds of $S^{n}$ and energy-minimizing maps from $\mathbf{R}^{2}$ to $S^{n}$. In particular, Corollary 4 restates a pivotal result from a previous paper on area-minimizing hypersurfaces [6, Theorem 1]. The latter result bears a very similar relationship to the regularity and Bernstein theorems in [6] for hypersurfaces, as Theorem 1 here bears to our present regularity and 
Liouville results. It seems a curious feature of this entire subject area that the more highly nonlinear theory of area minimization generally has preceded the very analogous, but relatively linear, theory of energy minimization.

Before we begin, we wish to thank R. Schoen for his interest in our work, and for helping us obtain the energy estimate of Lemma 2 below.

\section{A maximum principle}

Consider the (topological) product $N^{n}=L^{n-1} \times \mathbf{R}$, where $L$ is some Riemannian manifold. Let $\pi: L \times \mathbf{R} \rightarrow \mathbf{R}$ be the canonical projection. We establish in this section that for a certain class of metrics on $N$, any real-valued function $\pi \circ F$ on a Riemannian manifold $M^{k}$, which is obtained by projecting a harmonic map $F: M \rightarrow N$ into the $\mathbf{R}$ factor in $N$, satisfies an elliptic differential equation, and thereby a maximum principle.

The class of metrics we have in mind can be characterized as follows. There is a smooth function $w: N \rightarrow \mathbf{R}^{+}$such that, given an orthonormal basis $e_{1}, \cdots, e_{n-1}$ for $T_{P} L$ (the tangent space of $L$ at $p \in L$ ), and a unit vector $\nu \in T_{t} \mathbf{R}$, then at $(p, t) \in N$, the metric $\langle$,$\rangle satisfies$

$$
\left\langle e_{i}, e_{j}\right\rangle=\delta_{i j},\left\langle e_{i}, \nu\right\rangle=0,\langle\nu, \nu\rangle=w^{2}(p, t) .
$$

Here, $\delta_{i j}$ is the Kronecker delta, and $i, j=1, \cdots, n-1$. In a slight departure from more standard terminology (which would require $w$ to be independent of $t$ ), we will refer to this as a warped product metric on $N$, and write $N=L \times_{w} \mathbf{R}$ to denote the resulting Riemannian manifold.

Remark. It can be shown that, modulo certain diffeomorphisms $\phi: N \rightarrow N$ which satisfy $\pi \circ \phi=\pi$, this is exactly the class of metrics on $N$ which make each hypersurface $L \times\{t\}(t \in \mathbf{R})$, totally geodesic.

We may now introduce the elliptic equation mentioned above. The intrinsic divergence and gradient on $M$ will be denoted by $\operatorname{div}_{M}$ and $\operatorname{grad}_{M}$, respectively.

Lemma 1. Let $M, w, N$ be as above, let $F: M \rightarrow N$ be harmonic, and define $\boldsymbol{\theta}: M \rightarrow \mathbf{R}$ via $\boldsymbol{\theta}=\pi \circ F$. Then

$$
\operatorname{div}_{M}\left[w^{2} \operatorname{grad}_{M} \theta\right]-\frac{1}{2} \frac{\partial w^{2}}{\partial t}\left|\operatorname{grad}_{M} \theta\right|^{2}=0 .
$$

Proof. Since $N=L \times{ }_{w} \mathbf{R}$, there exists a map $f: M \rightarrow L$ such that $F=(f, \theta)$. An easy calculation then shows that the energy density $|D F|^{2}$ decomposes as follows:

$$
|D F|^{2}=|D f|^{2}+w^{2}\left|\operatorname{grad}_{M} \theta\right|^{2}
$$


At the same time, for any compactly supported test function $\phi: M \rightarrow \mathbf{R}$, there is a variation extending $\theta$, and thereby $F$, to $M \times(-1,1)$ in such a way that for each $(x, s) \in M \times(-1,1)$,

$$
F(x, s)=(f(x), \theta(x, s)), \quad \dot{\theta}:=\left.\frac{\partial \theta}{\partial s}\right|_{s=0}=\varphi .
$$

A standard first variation argument based on the harmonicity of $F$ consequently yields the identity

$$
0=\int_{M} \dot{\theta} \frac{\partial w^{2}}{\partial t}\left|\operatorname{grad}_{M} \theta\right|^{2}+2 w^{2}\left\langle\operatorname{grad}_{M} \theta, \operatorname{grad}_{M} \dot{\theta}\right\rangle d v_{M}
$$

But $\dot{\boldsymbol{\theta}}=\varphi$, and since $\partial / \partial s$ commutes with differentiation on $M$, we may integrate by parts to obtain

$$
0=\int_{M} \phi \cdot\left[\operatorname{div}_{M}\left(w^{2} \operatorname{grad}_{M} \theta\right)-\frac{1}{2} \frac{\partial w^{2}}{\partial t}\left|\operatorname{grad}_{M} \theta\right|^{2}\right] d v_{M}
$$

In the usual way, the fact that this holds for arbitrary $\phi$ now implies the truth of Lemma 1. q.e.d.

Combining this lemma in an obvious way with the strong maximum principle, we obtain a useful corollary.

Corollary 1. If $M$ is compact, then $F(M)$ lies in $L \times\{t\}$ for some $t \in \mathbf{R}$.

We end this section with an application of these ideas. Consider a harmonic map $F: M \rightarrow \bar{N}$, where some covering space of $\bar{N}$ is assumed to admit a warped product metric. The following corollary is then immediate, by passage to a map between universal covers.

Corollary 2. If $\bar{N}$ is covered by a warped product, $M$ is compact, and $\pi_{1}(M)$ is finite, then the image of any harmonic map $F: M \rightarrow \bar{N}$ lies in a totally geodesic hypersurface of $\bar{N}$.

\section{The Linking Theorem}

This section and the next are concerned with harmonic maps to the unit sphere $S^{n} \subset \mathbf{R}^{n+1}$. Our previous results will come into play here because of the following key observation.

Observation 1. If $\Sigma^{n-2} \subset S^{n}$ is any totally geodesic, codimension two subsphere, then $S^{n} \sim \Sigma$ (the complement of $\Sigma$ in $S^{n}$ ) is isometric to a warped product.

To see this, first rotate $S^{n} \sim \Sigma$ so that

$$
\Sigma=S^{n} \cap\left\{\left(x_{1}, \cdots, x_{n+1}\right) \in \mathbf{R}^{n+1}: x_{1}^{2}+x_{2}^{2}=0\right\} .
$$


Denoting by $S_{+}^{n-1} \subset \mathbf{R}^{n}$ the $(n-1)$-dimensional open upper hemisphere,

$$
S_{+}^{n-1}=\left\{\left(y_{1}, \cdots, y_{n}\right) \in \mathbf{R}^{n}: y_{1}^{2}+\cdots+y_{n}^{2}=1, y_{n}>0\right\},
$$

we then have

$$
S^{n} \sim \Sigma=S_{+}^{n-1} \times{ }_{w} S^{1}
$$

where

$$
w^{2}\left(y_{1}, \cdots, y_{n}, \theta\right)=y_{n}^{2} .
$$

(Here $\theta$ denotes a point in $S^{1}$.) Indeed, the map which sends

$$
\left(y_{1}, \cdots, y_{n}, \theta\right) \in S_{+}^{n-1} \times S^{1}
$$

to

$$
\left(y_{n} \cos (\theta), y_{n} \sin (\theta), y_{1}, \cdots, y_{n-1}\right) \in S^{n} \subset \mathbf{R}^{n+1},
$$

is easily seen to be an isometry.

The terminology of our next theorem is as follows. A map $F: M \rightarrow S^{n}$ will be said to meet $\Sigma$ if $F(M) \cap \Sigma \neq \varnothing$. If $F$ does not meet $\Sigma$, but as a map to $S^{n} \sim \Sigma$ it is homotopically nontrivial, we will say $F$ links $\Sigma$. Note that this definition extends the usual, homological notion of "linking with $\Sigma$ ", for immersions $F: M^{1} \rightarrow S^{n} \sim \Sigma$.

Theorem 1. Let $F: M \rightarrow S^{n}$ be a nonconstant harmonic map on a compact manifold $M$. Then $F$ either links or meets $\Sigma$.

Proof. We will show that if $F$ neither meets nor links $\Sigma, F$ is constant. Indeed, in this situation, $F$ is a null-homotopic harmonic map to $S_{+}^{n-1} \times{ }_{w} S^{1}$, and such a map necessarily lifts to a harmonic map

$$
\tilde{F}: M \rightarrow S_{+}^{n+1} \times{ }_{w} \mathbf{R},
$$

because the latter target is universal cover to the former. By Corollary 1 , it then follows that $\tilde{F}(M) \subset S_{+}^{n-1} \times\{t\}$ for some $t \in \mathbf{R}$, hence $F(M) \subset S_{+}^{n-1} \times\{\theta\}$ for some $\theta \in S^{1}$. In other words, we may view $F$ as a map to $S_{+}^{n+1}$. But it is well-known that a harmonic map from a compact manifold to an open hemisphere is always constant. In fact, going back to our characterization of $S_{+}^{n-1}$ as the upper hemisphere of $S^{n-1} \subset \mathbf{R}^{n}$, we recall [3] that harmonicity of $F: M \rightarrow S^{n-1}$ is equivalent to the Euler-Lagrange system

$$
\left(\Delta_{M}+|D F|^{2}\right) F_{i}=0, \quad i=1, \cdots, n
$$

Here $\Delta_{M}$ denotes the intrinsic Laplace operator of $M$, and $F_{i}=y_{i} \circ F$. Since $F_{n}>0$, set $i=n$ and integrate the associated Euler-Lagrange equation over $M$. $|D F|^{2}$ is thereby forced to vanish identically, and $F$ is constant. This proves Theorem 1. q.e.d. 
We next wish to insert a simple topological observation which will soon play a useful role. Note that $S_{+}^{n-1} \times S^{1}$ is homotopy equivalent to $S^{1}$. Hence a map $F: M \rightarrow S_{+}^{n-1} \times S^{1}$ is null-homotopic if and only if its projection onto the $S^{1}$ factor is null-homotopic. But homotopy classes of maps $M \rightarrow S^{1}$ form a group, (commonly known as the first cohomotopy group of $M$, and denoted by $\pi^{1}(M)$ ), which is canonically isomorphic to the first integral cohomology group $H^{1}(M)$. (The isomorphism is induced by pulling back a generator of $H^{1}\left(S^{1}\right)$.) We therefore have the following.

Observation 2. If $H^{1}(M)=0$, then all maps $F: M \rightarrow S^{n} \sim \Sigma$ are null-homotopic, i.e. fail to link $\Sigma$.

We conclude this section with two corollaries regarding minimal submanifolds of the sphere.

Corollary 3. A compact minimal $M^{k} \subset S^{n}$ either links or meets every great $\Sigma^{n-2} \subset S^{n}$. If $H^{1}(M)=0, M$ meets every $\Sigma$.

Proof. Combine Theorem 1, Observation 2, and the well-known harmonicity of minimal immersions. q.e.d.

The above corollary should be compared with the familiar fact that any minimal $M^{k} \subset S^{n}$ meets every great $S^{n-1} \subset S^{n}$. (Indeed, since $M$ is harmonically immersed, its center of mass must reside at the origin in $\mathbf{R}^{n+1}$.) The corollary below, on the other hand, restates a key result from our earlier paper [6, Theorem 1]. In fact, the present work has its beginnings in our efforts to find a more illuminating proof of that theorem, which now follows easily.

Corollary 4 ([6]). Let $M \subset S^{n}$ be a compact orientable minimal hypersurface $(k=n-1)$, with $H^{1}(M)=0$. If the Gauss image of $M$ in $S^{n}$ omits $\Sigma$, then $M$ is an equator.

Proof. Combine Theorem 1, Observation 2, and the well-known harmonicity of the Gauss map on $M$ (i.e., the restriction to $M$ of the Gauss map on the central cone over $M$ in $\mathbf{R}^{n+1}$ ).

\section{Regularity}

Given our linking theorem in the previous section, we can easily exploit the theory of Schoen \& Uhlenbeck to obtain a regularity theorem for energyminimizing maps to $S^{n} \sim \Sigma$.

Theorem 2. An energy-minimizing map $F: M \rightarrow S^{n}$, which omits a neighborhood of a codimension-two great subsphere $\Sigma \subset S^{n}$, is everywhere smooth.

Proof. Recall that for any energy-minimizing map $F: M \rightarrow N$ between arbitrary Riemannian manifolds, the codimension of $\operatorname{sing}(F) \subset M$ is at least 3, and that this lower bound can be raised by information concerning $N$ alone. 
Indeed, to paraphrase Theorem IV of [7], if for some $k \geqslant 1$, and each $d=$ $1, \cdots, k$, every smooth harmonic map $f: S^{d+1} \rightarrow N$ is constant, then $\operatorname{sing}(F) \subset M$ has codimension at least $3+k$. In our present situation, we may apply this fact with $N=S^{n} \sim \Sigma$. Since $H^{1}\left(S^{d+1}\right)=0$ for every positive integer $d$, the desired result now follows immediately from Theorem 1 and Observation 2 of the preceding section. q.e.d.

Actually, the above proof only argues a special case. To see this, we return to the warped product setting of $\$ 1$. Consider any Riemannian manifold $N$ having a Riemannian covering space $\tilde{N}$ which admits a warped product decomposition; say, $\tilde{N}=L \times{ }_{w} \mathbf{R}$. Since $S^{d+1}$ is simply connected for every $d \geqslant 1$, each smooth harmonic map $f: S^{d+1} \rightarrow N$ lifts to a harmonic map $\tilde{f}$ : $S^{d+1} \rightarrow \tilde{N}$, which in turn factors through a harmonic map $\bar{f}: S^{d+1} \rightarrow L$, by Corollary 1 . The general version of Theorem 2 is therefore given as follows.

Theorem 3. If $M, L, N$, and $\tilde{N}$ are as above, $k \geqslant 1$, and for every $d=1, \cdots, k$, each harmonic map $f: S^{d+1} \rightarrow L$ is constant, then the singular set of any energy-minimizing map $F: M \rightarrow N$ has codimension at least $3+k$.

Finally, we state another special case.

Corollary 5. If $L$ has negative sectional curvature, or is strictly geodesically convex, then every energy-minimizing map $F: M \rightarrow L \times_{w} \mathbf{R}$ is everywhere smooth.

Proof. The required information about harmonic maps from spheres into $L$ is given in the Corollary on p. 310 of [7], based on well-known results of Eells \& Sampson [4] (in the negative curvature case), and Hildebrandt, Kaul \& Widman [5] (in the strictly convex case).

\section{Liouville theorem}

In this section, we will establish a Liouville theorem for energy-minimizing maps $F: \mathbf{R}^{k} \rightarrow S^{n}$ which are bounded away from a codimension-two, totally geodesic subsphere $\Sigma \subset S^{n}$. First, we require an energy growth estimate. Let $B_{r}^{k}$ (or simply $B_{r}$, when the dimension is clear from context), denote the open ball of radius $r$, centered at the origin in $\mathbf{R}^{k}$.

Lemma 2. If $F: \mathbf{R}^{k} \rightarrow S^{n}$ is an energy-minimizing map which omits a neighborhood of some point $p \in S^{n}$, then

$$
\int_{B_{r}^{k}}|D F|^{2} d x \leqslant C r^{k-2}
$$

for all $r>0$. Here $C=C(n, k)$ is a constant.

Remark. The main content of this lemma resides in the case $n=2$; it holds when $n>2$ even without the omitted neighborhood assumption (cf. proof of $[8$, Theorem 2.7]). 
Proof. When $k=1$ or $n=1$, the lemma holds trivially because $|D F|^{2}$ vanishes identically. We leave this as an exercise for the reader and proceed under the assumption $k, n>1$.

In this case, by using exponential polar coordinates centered at the point $q \in S^{n}$ antipodal to $p$, we may consider $F$ as a map into $B_{\pi}^{n}$. But for any map $G \in \mathbf{L}_{1}^{2}\left(B_{r}^{k}, B_{\pi}^{n}\right)$, the energy density $|D G|^{2}$ can be measured relative to either the flat metric (induced by the inclusion $B_{\pi}^{n} \subset \mathbf{R}^{n}$ ), or the spherical metric (which makes $B_{\pi}^{n}$ isometric to $S^{n} \sim\{p\}$ ). Precisely, we have

$$
\begin{gathered}
|D G|^{2}=\left(\frac{\partial G}{\partial r}\right)^{2}+\left|D_{T} G\right|^{2} \quad \text { (flat metric), } \\
|D G|^{2}=\left(\frac{\partial G}{\partial r}\right)^{2}+\frac{\sin ^{2} r}{r^{2}}\left|D_{T} G\right|^{2} \quad \text { (spherical metric) }
\end{gathered}
$$

where the "tangential" (as opposed to radial) energy $\left|D_{T} G\right|^{2}$ is, in fact, defined by the first of these equations. Correspondingly, we obtain two values for the energy integral

$$
\int_{B_{r}^{k}}|D G|^{2} d x
$$

which we will denote by

$$
\mathbf{E}_{r}(G) \quad \text { (flat metric), } \quad \tilde{\mathbf{E}}_{r}(G) \quad \text { (spherical metric), }
$$

for the rest of this section. Since the particular map $F$ being considered here omits a full neighborhood of $p \in S^{n}$, we actually have a map to $B_{\pi-\delta}^{n}$ for some strictly positive $\delta>0$, and consequently

$$
\tilde{\mathbf{E}}_{r}(F) \leqslant \mathbf{E}_{r}(F) \leqslant C \tilde{\mathbf{E}}_{r}(F)
$$

for some constant $C=C(\delta)$. Thus, whereas $F$ minimizes $\tilde{\mathbf{E}}$, it will be useful to compare it, given $r>0$, with the harmonic map $H_{r} \in \mathbf{L}_{1}^{2}\left(B_{r}^{k}, B_{\pi-\delta}^{n}\right)$ which agrees with $F$ on $\partial B_{r}^{k}$, and minimizes $\mathbf{E}$. The existence of $H_{r}$ for almost all $r>0$ is guaranteed by Fubini's Theorem and the convex hull property for harmonic maps to $\mathbf{R}^{n}$.

Observe that each coordinate function of $H_{r}$ is itself harmonic, hence may be expanded in terms of spherical harmonics on $\mathbf{R}^{k}$. Using this expansion, together with Green's Theorem and the Schwarz inequality, it is then a straightforward matter to deduce the estimate

$$
\mathbf{E}_{r}\left(H_{r}\right) \leqslant C\left(\int_{\partial B_{r}}\left|H_{r}\right|^{2} d x\right)^{1 / 2}\left(\int_{\partial B_{r}}\left|D_{T} H_{r}\right|^{2} d x\right)^{1 / 2},
$$


for some $C=C(n, k)$. But $\left|D_{T} H_{r}\right|^{2}=\left|D_{T} F\right|^{2}$, and we have $\left|H_{r}\right|^{2} \leqslant(\pi-\delta)^{2}$, so that

$$
\mathbf{E}_{r}(F) \leqslant C \tilde{\mathbf{E}}_{r}(F) \leqslant C \tilde{\mathbf{E}}_{r}\left(H_{r}\right) \leqslant C \mathbf{E}_{r}\left(H_{r}\right),
$$

hence

$$
\mathbf{E}_{r}(F) \leqslant C(\pi-\delta)\left(r^{k-1}\right)^{1 / 2}\left(\int_{\partial B_{r}}|D F|^{2}: d x\right)^{1 / 2} .
$$

Squaring this relation, we then obtain the following differential inequality, with $C$ independent of $F$ :

$$
\mathbf{E}_{r}^{2}(F) \leqslant C r^{k-1} \frac{d}{d r} \mathbf{E}_{r}(F) .
$$

That is, unless $\mathbf{E}_{r}(F)$ vanishes identically, we have

$$
-\frac{d}{d r}\left(\frac{1}{\mathbf{E}}\right) \geqslant \begin{cases}\frac{C}{2-k} \frac{d}{d r} r^{2-k}, & (k>2), \\ C \frac{d}{d r} \ln (r), & (k=2) .\end{cases}
$$

For $k>2$, we then obtain our lemma by integrating the above inequality from $r$ to infinity, observing that by the monotonicity formula for energy, [7, Proposition 2.4], $\mathbf{E}_{R}(F) \rightarrow \infty$ as $R \rightarrow \infty$.

When $k=2$, a similar integration, from say, $r$ to $R$, leads to the apparent conundrum

$$
\frac{1}{\mathbf{E}_{r}(F)} \geqslant \frac{1}{\mathbf{E}_{r}(F)}-\frac{1}{\mathbf{E}_{R}(F)} \geqslant C \ln \left(\frac{R}{r}\right) .
$$

The contradiction here as $R \rightarrow \infty$ forces us to conclude that, contrary to our earlier assumption, $\mathbf{E}_{r}(F)$ vanishes identically. In other words, Lemma 2 holds trivially in this final case, and its proof is complete. q.e.d.

With out last argument (for $k=2$ ), we have established more than just the statement of the lemma. Indeed, thinking of $S^{n}$ as the "Riemann sphere", with $p$ as the point at infinity, we obtain a precise analog of the classical holomorphic Liouville theorem, for energy minimizing maps.

Corollary 6. Let $F: \mathbf{R}^{2} \rightarrow S^{n}$ be an energy-minimizing map which is bounded away from some point in $S^{n}$. Then $F$ is constant.

Note that every harmonic function on $\mathbf{R}^{2}$, reduced modulo $2 \pi$, gives rise to a harmonic map $\mathbf{R}^{2} \rightarrow S^{2}$ whose image is the equator. Corollary 6 is thus clearly false for maps which are stationary, but not minima.

We can now give the statement and proof of our main Liouville theorem. Again, $\Sigma \subset S^{n}$ is a codimension-two, totally geodesic subsphere. 
Theorem 4. When $n>1$, any energy-minimizing map $F: \mathbf{R}^{k} \rightarrow S^{n}$ which is bounded away from $\Sigma$, is constant.

Proof. Since $n>1$ and $F$ omits a neighborhood of $\Sigma, F$ omits a neighborhood of a point. Consequently, by combining Lemma 2 with the degree $k-2$ homogeneity of $\mathbf{E}$ under scaling, we conclude that the maps

$$
F_{j}: B_{1}^{k} \rightarrow S^{n} \sim \Sigma
$$

defined by $F_{j}(x)=F(j x)$ for $x \in B_{1}^{k}, j=1,2, \cdots$, are uniformly bounded in energy, hence in $\mathbf{L}_{1}^{2}$. It is therefore possible to extract a weakly convergent subsequence $\left\{F_{j}\right\}$ tending to a limit $F_{\infty}$, and by [7, 4.6], $F_{\infty}$ is actually a strong limit in $\mathbf{L}_{1}^{2}$. It follows that $F_{\infty}$ omits a neighborhood of $\Sigma$, and via the argument of [7, 4.7], that $F_{\infty}$ is weakly harmonic (though not necessarily minimizing), as well as radially homogeneous (i.e., the radial derivative of $F_{\infty}$ vanishes a.e. on $B_{1}^{k}$ ).

$F_{\infty}$ is therefore precisely the type of map one obtains by "blowing up" an energy-minimizing map at an interior point of its domain (cf. [7, 4.7]). The dimension reducing technique of $[7, \S 5]$ thus applies in our setting, and $F_{\infty}$ is subject to the same regularity criterion we saw in the previous section. Namely, since every smooth harmonic map $f: S^{d+1} \rightarrow S^{n} \sim \Sigma$ is known to be constant, $F_{\infty}$ is necessarily smooth. Being radially homogeneous too, $F_{\infty}$ is in fact constant.

This, finally, implies the constancy of $F$ itself. For by the monotonicity formula for energy, [7, 2.4], our $\mathbf{L}_{1}^{2}$-convergent sequence $F_{j} \rightarrow F_{\infty}$ has nondecreasing energy, whereas, by the above, $\left|D F_{\infty}\right|^{2}=0$.

\section{Appendix on nodal sets}

Here we make some observations which relate the behavior described in $\S 2$ for harmonic maps $M \rightarrow S^{n}$, to the nodal sets of eigenfunctions for a Schrodinger operator on a compact Riemannian manifold $M$. A Schrodinger operator on $M$ is a differential operator of the form $\Delta_{M}+V$, where $V$ is a smooth potential function. The nodal set of a function $f: M \rightarrow R$ is the preimage $f^{-1}(0) \subset M$.

Nodal sets of eigenfunctions are intrinsic geometric objects on a Riemannian manifold, but very little is known about them, even when $V$ is constant, i.e. an eigenvalue of the Laplacian. Several open questions are discussed in Yau's Problem Section [9], and some basic results on nodal sets are proved in a paper of Cheng [1], especially for two-dimensional $M$. In fact, Lemma 4.2 of Cheng's paper gives a (rather special) condition which forces the nodal sets of two 
linearly independent eigenfunctions to intersect each other. Under a much weaker, more natural assumption, we draw precisely the same conclusion in Theorem 5 below.

Using Theorem 5, the reader should find it easy to fashion an alternate, and very geometric, proof of our linking theorem (Theorem $1, \S 2$ ). Indeed, simply rotate $S^{n} \sim \Sigma$ as in Observation $1, \S 2$, and àpply Theorem 5 to the functions $F_{1}, F_{2}$, appearing in the Euler-Lagrange system for the harmonic map $F$ (see proof of Theorem 1).

In the following, $N_{f}$ will denote the nodal set of a function $f: M \rightarrow \mathbf{R}$, and by a nodal domain of $f$, we shall mean a connected component of $M \sim N_{f}$.

Theorem 5. Let $u, v: M \rightarrow \mathbf{R}$ be nontrivial solutions of a Schrodinger operator on a compact Riemannian manifold $M$, and assume $H^{1}(M)=0$. Then

$$
N_{u} \cap N_{v}=\varnothing \quad \text { iff } \quad N_{u} \cup N_{v}=\varnothing .
$$

Remark. This result fails when $H^{1}(M)$ does not vanish. For instance, consider the nodal sets of solutions to $\left(\Delta_{M}+1\right) u=0$ when $M=S^{1}$.

Proof. Assume that $N_{u} \cap N_{v}=\varnothing$. Then $u^{2}+v^{2}>0$ on $M$, and we may form the map

$$
\theta=\frac{(u, v)}{\sqrt{u^{2}+v^{2}}}: M \rightarrow S^{1} \subset \mathbf{R}^{2} .
$$

Since $H^{1}(M)=0, \theta$ is null-homotopic as a map to $S^{1}$, hence lifts to a real-valued function, call it $\tilde{\theta}$, such that

$$
\theta=(\cos \tilde{\theta}, \sin \tilde{\theta}) \text {. }
$$

Letting

$$
\begin{aligned}
Z & =\{n \pi / 2 \in \mathbf{R}: n \in \mathbf{Z}\}, \\
y^{+} & =\max _{M} \tilde{\theta}, \quad y^{-}=\min _{M} \tilde{\theta},
\end{aligned}
$$

we first observe that if $y^{+} \in Z$, then for any sufficiently small neighborhood of a point $p \in M$ such that $\tilde{\theta}(p)=y^{+}$, either $u$ or $v$ would vanish, but not change sign. This is impossible by a well-known argument based on the Hopf boundary point lemma; we must conclude that $y^{+} \notin Z$. Similarly for $y^{-}$. Thus, denoting by $I$ the connected component of $\mathbf{R} \sim Z$ which contains $y^{+}$, we see that the preimage of $I$ under $\tilde{\theta}$ must consist of nodal domains for one of the solutions $u$ or $v$. It follows that $\tilde{\theta}(M)$ is actually contained in $I$, for otherwise these nodal domains of one solution would all be properly contained in nodal domains of the other solution. It is a classical fact that such "concentric" nodal domains cannot occur (see [2, p. 458], for example). Consequently, neither $u$ nor $v$ has any zero, and the proof of Theorem 5 is complete. 


\section{References}

[1] S. Y. Cheng, Eigenfunctions and nodal sets, Comment. Math. Helv. 51 (1976) 43-55.

[2] R. Courant \& D. Hilbert, Methods of mathematical physics, Vol. 1, Interscience, New York, 1953.

[3] J. Eells \& L. Lemaire, A report on harmonic maps, Bull. London Math. Soc. 10 (1978) 1-68.

[4] J. Eells \& J. H. Sampson, Harmonic mappings of Riemannian manifolds, Amer. J. Math. 86 (1964) 109-160.

[5] S. Hildebrandt, H. Kaul \& K. O. Widman, An existence theorem for harmonic mappings of Riemannian manifolds, Acta Math. 138 (1970) 550-569.

[6] B. Solomon, On the Gauss map of an area-minimizing hypersurface, J. Differential Geometry 19 (1984) 221-232.

[7] R. Schoen \& K. Uhlenbeck, A regularity theory for harmonic maps, J. Differential Geometry 17 (1982) 307-335.

[8] __ Regularity of minimizing harmonic maps into the sphere, to appear in Invent. Math.

[9] S. T. Yau, Problem section, Seminar in Differential Geometry, Annals of Math. Studies, No. 102, Princeton University Press, Princeton, NJ, 1982.

INDIANA UNIVERSITY 